\title{
Measurements of Inclined Air Showers with the Auger Engineering Radio Array at the Pierre Auger Observatory
}

\author{
Marvin Gottowik $^{* a}$ for the Pierre Auger Collaboration ${ }^{\dagger b}$ \\ ${ }^{a}$ Bergische Universität Wuppertal, Gaußstraße 20, 42097 Wuppertal, Germany \\ ${ }^{b}$ Observatorio Pierre Auger, Av. San Martín Norte 304, 5613 Malargüe, Argentina \\ E-mail: auger_spokespersons@fnal.gov \\ Full author list: http://www.auger.org/archive/authors_icrc_2019.html
}

\begin{abstract}
The Auger Engineering Radio Array (AERA) comprises 153 autonomous radio antenna stations distributed over an area of $17 \mathrm{~km}^{2}$. It is operated in coincidence with the surface detector array and fluorescence telescopes of the Pierre Auger Observatory so that hybrid observations of extensive air showers initiated by cosmic rays in the EeV energy range can be performed. A first analysis of the radio emission of more than 500 inclined air showers with zenith angles between $60^{\circ}$ and $84^{\circ}$ was published last year. Here, we provide an update of the analysis including recent data, approximately quadrupling the event statistics. For inclined showers, the radio signal is found to be distributed over an area of several $\mathrm{km}^{2}$, allowing air showers to be measured with radio antennas on a grid as sparse as the $1500 \mathrm{~m}$ spacing of the 1600 water-Cherenkov Detector (WCD) stations. For a subset of these inclined events, for which the primary energy can be reconstructed with the data from the WCD array, we verify that the measured radio amplitudes agree with Monte Carlo simulations made using CoREAS. Special challenges of a radio event reconstruction dedicated to inclined air showers will also be discussed.
\end{abstract}

36th International Cosmic Ray Conference - ICRC2019

24 July - 1 August, 2019

Madison, Wisconsin, USA

\footnotetext{
${ }^{*}$ Speaker.

${ }^{\dagger}$ for collaboration list see PoS(ICRC2019)1177
} 


\section{Introduction}

Ultrahigh energy cosmic rays interact with the Earth's atmosphere and produce an extensive air shower of secondary particles. The detection of air showers using the emitted radio signals has become a standard technique for modern cosmic ray experiments and yields a resolution in the reconstruction of important air shower observables that is competitive with particle and fluorescence detectors [1,2]. Radio detection combines the advantages of originating from the well-understood electromagnetic component of the air shower, being sensitive to the longitudinal shower profile and having an operational time of nearly $100 \%$.

As the radio emission is strongly forward-beamed it only illuminates areas with a diameter of a few hundred meters on the ground in the case of vertical showers. This requires a dense antenna grid. However, for inclined air showers the shower develops geometrically more distant to the ground. As there is no absorption or scattering of the emitted radio signal in the atmosphere it is expected that an area of several $\mathrm{km}^{2}$ on the ground is illuminated [3]. This would enable measurements of inclined air showers with a sparse antenna array.

\section{The Auger Engineering Radio Array}

The Pierre Auger Observatory is a hybrid detector for the measurement of ultra high energy cosmic rays located in Mendoza Province, Argentina [4]. Its baseline detectors comprise the world's largest surface detector (SD) array of 1660 water Cherenkov particle detectors covering in total an area of $3000 \mathrm{~km}^{2}$, and a fluorescence detector (FD) overlooking the array from 4 sites with 27 telescopes. The Auger Engineering Radio Array (AERA) [5] is situated in the northwestern part within the $750 \mathrm{~m}$ spaced SD array and in the field of view of the Coihueco and HEAT FD telescopes.

AERA is an engineering array, hence different antenna types, electronics and spacings of the stations have been tested. The deployment was done in three phases starting in summer 2011, the current layout (AERA III) was finalized in March 2015. Today, AERA comprises 153 autonomous radio stations spread over an area of $\sim 17 \mathrm{~km}^{2}$. In Fig. 1, a map of AERA is shown and the individual deployment phases are indicated. For the dense center of AERA (phase I) 24 logarithmic periodic dipole antennas (LPDA) were deployed on a $144 \mathrm{~m}$ grid. For the later phases butterfly antennas were installed with spacings of $250 \mathrm{~m}, 375 \mathrm{~m}$ and $750 \mathrm{~m}$. Each radio station consists of two perpendicularly oriented antennas, aligned with magnetic north and east, which are sensitive to the radio emission in the frequency range from 30 to $80 \mathrm{MHz}$.

\section{Analysis of radio emission from inclined air showers}

A previous analysis using 561 air showers (1.5 years of AERA phase II data) has shown the potential of a detection of those inclined air showers, even with a sparse antenna grid, at $1.5 \mathrm{~km}$ distances like the SD array [6]. Here, we update that analysis including four more years of AERA phase III data. Furthermore, the CoREAS simulation code [7] has been optimized for inclined air showers and now yields more precise results.

For the particle data we use the standard Auger reconstruction of inclined air showers [8]. For the radio detection we require at least three antenna stations that detected a radio pulse with a 


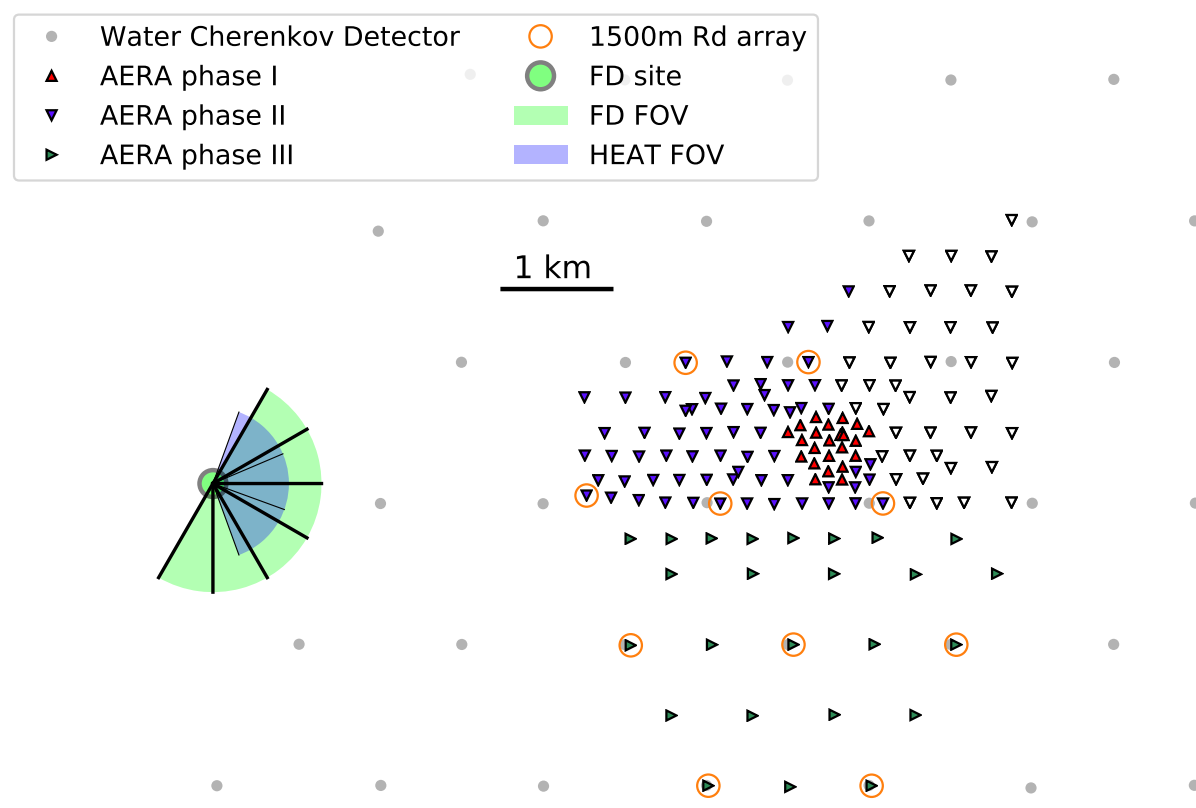

Figure 1: Map of AERA within the Pierre Auger Observatory. The orientation of the triangles indicate the three expansion phases of AERA. The open triangles denote stations that are not capable of receiving an external trigger from the surface detector array and are therefore excluded in this analysis. The orange circles highlight radio stations that will be used for the sparse radio grid in section 3.4.

signal-to-noise ratio ${ }^{1}$ above 10 . The direction is obtained by a plane-wave fit to the arrival times of the radio pulses. We fit a Gumbel function to the distribution of the opening angle between radio and particle detector reconstruction and only select showers within the $3 \sigma$ interval around the mean value (cf. Fig. 2). This evaluates to a maximum opening angle of $2.6^{\circ}$. In total we obtain 1972 events in the time period of $26.06 .2013-8.3 .2019$ with zenith angles between $60^{\circ}$ and $84^{\circ}$. We do not extend the analysis to showers above $84^{\circ}$ zenith angle as this requires additional studies of the surface detector reconstruction.

\subsection{Characterization of total data set}

In the following, we use the arrival direction as reconstructed with the SD. The azimuth distribution (not shown here) shows a clear north-south asymmetry, as showers coming from south have a stronger geomagnetic emission and thus are easier to detect compared to showers coming from the north. The distribution of the $\sin ^{2}$ of the zenith angle $\theta$ increases for more inclined showers (cf. Fig. 2) which indicates an increasing detection efficiency for the coincident observation of air showers with the SD and AERA.

\footnotetext{
${ }^{1}$ Squared maximum of the electric-field amplitude after projection onto the ground plane divided by squared RMS of the background electric-field amplitudes
} 

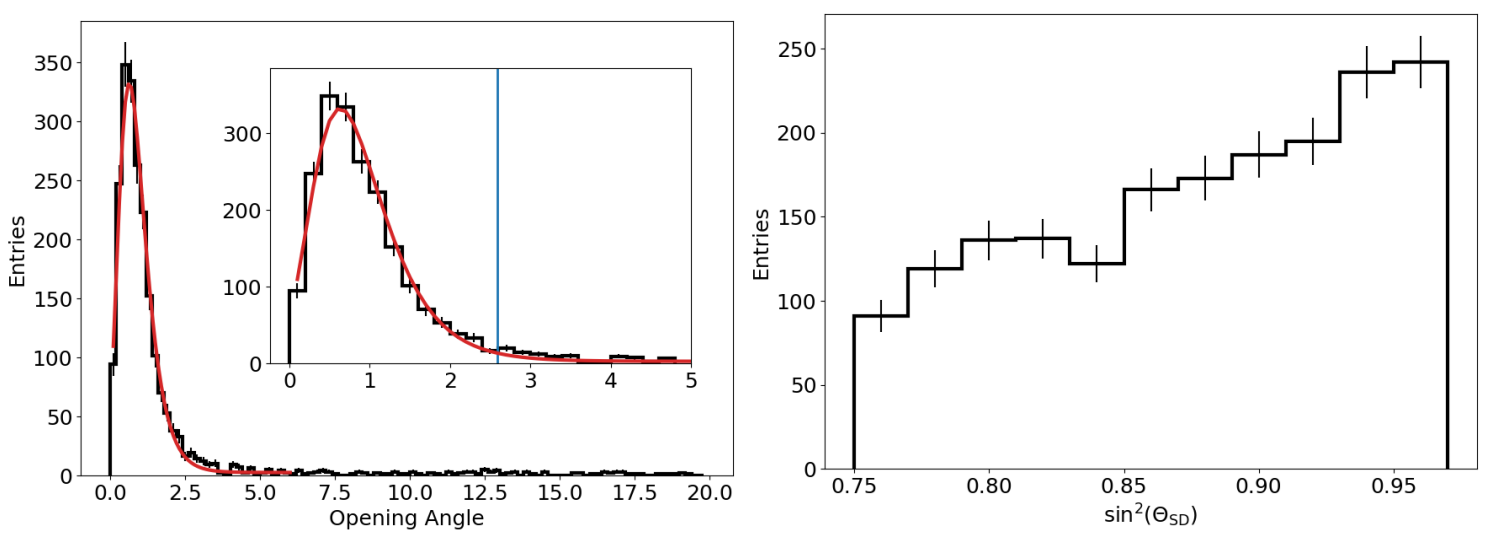

Figure 2: Left: Distribution of the opening angle of particle and radio shower axis. A Gumbel fit is shown in red. The inset figure shows a zoom in on the signal peak, the maximum opening angle used in this analysis, corresponding to a $3 \sigma$ deviation from the mean, is indicated by the vertical line. Right: Distribution of the $\sin ^{2}$ of the zenith angles. For each bin, Poissonian errors are shown.

\subsection{Size of the radio footprint on ground}

To estimate the increase of the radio footprint in the shower plane, excluding projection effects to the ground, we calculate the maximum distance of all antennas with a detected signal from the shower axis reconstructed from particle data. Fig. 3 shows a clear increase of this distance with increasing zenith angle. Qualitatively, this is in agreement with the assumption of forward-beamed radio emission without absorption and scattering in the atmosphere.

A particularly interesting event is shown in Fig. 4. The shower core, as reconstructed by the surface detector, is approximately $12 \mathrm{~km}$ away from AERA. In total 14 radio stations have a measured signal clearly above noise. The radio direction reconstruction agrees within $1.2^{\circ}$ to the reconstruction of the particle detector. This example shows, that for very inclined air showers, the radio footprint can be significantly larger than the "particle footprint" on the ground.

\subsection{Comparison with CoREAS simulations}

For this analysis we adapt the standard selection of events with a proper reconstructed energy by the surface detector. Normally the zenith angle is limited to $80^{\circ}$, here we extend the analysis up to $84^{\circ}$ and lower the energy threshold to $10^{18.5} \mathrm{eV}$. This results in a degradation in the reconstruction performance that is not relevant for this analysis, as the energy determination remains bias-free and has a resolution better than 25\% [9] (19.3\% for the standard SD event selection of inclined air showers [8], further details in [6]). This subselection yields 170 events. The energy distribution of this subset is shown in Fig. 5, reaching up to $10^{19.8} \mathrm{eV}$.

For each event we perform one full Monte Carlo Simulation with CoREAS using the reconstruction of the surface detector as input. We only simulate proton primaries to obtain maximal shower-to-shower fluctuations, the impact of heavier primary particles is below $10 \%$ and thus smaller than the uncertainty of the energy scale and calibration [6]. We perform a detailed radio detector simulation for each simulated shower, add measured noise and analyse the simulations in the same way as the measured events. A direct comparison of the amplitudes for each antenna 


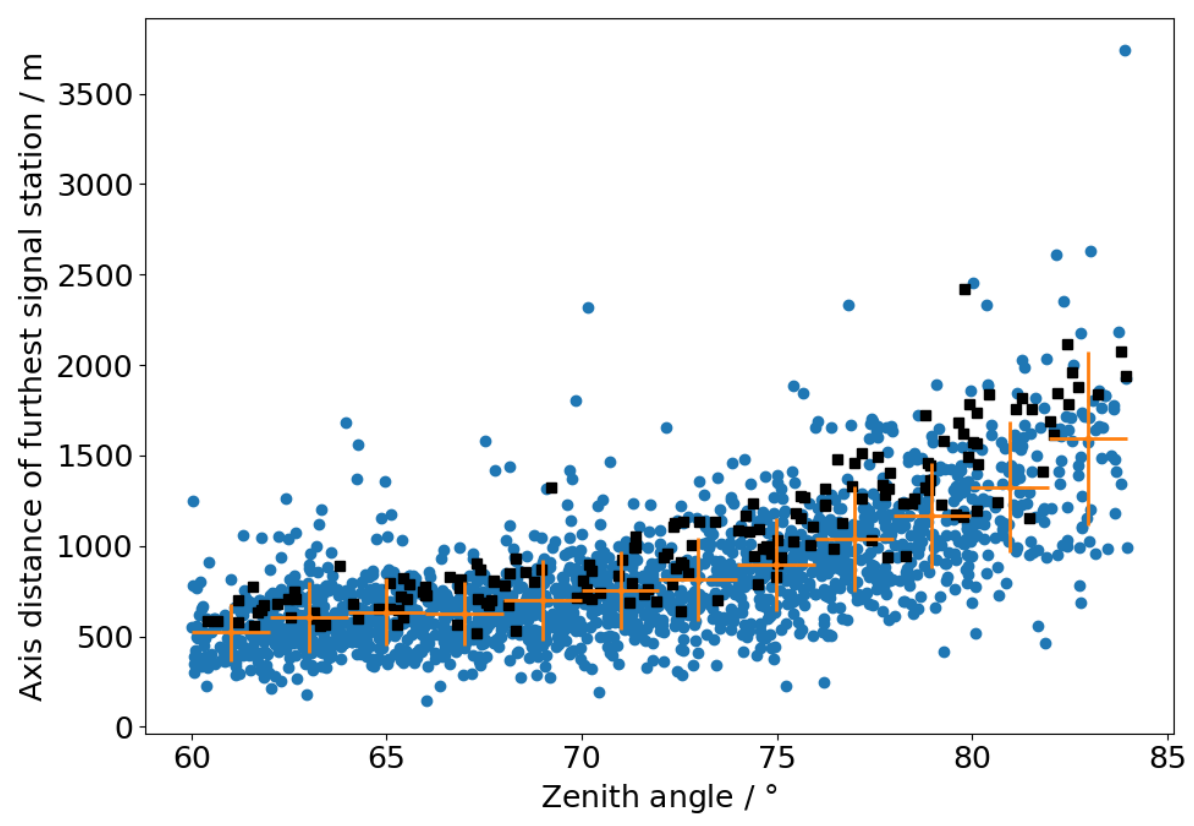

Figure 3: Maximum axis distance of a radio station with a detected signal above noise as a function of zenith angle. Black squares represent the events that have a reconstructed energy from the surface detector (see section 3.3), the remaining events are shown as blue circles. The profile is added in orange, binned in $2^{\circ}$ zenith angle.

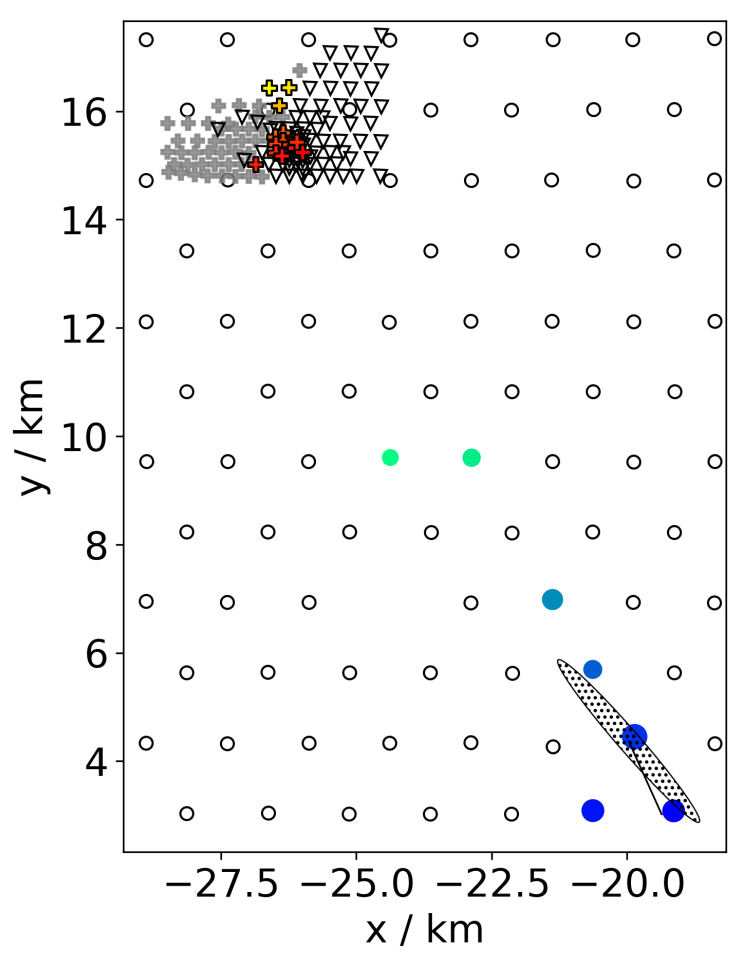

Figure 4: Visualization of the event with the very large radio footprint. The colored circles indicate signals measured with the surface detector, color indicating arrival time and size encoding deposited energy per station. The AERA stations with a detected signal are marked as colored pluses, color and size denote arrival time and energy fluence, respectively. Gray pluses represent stations that did not detect a signal clearly above noise. The open triangles are stations which are not used in this analysis. The impact point reconstructed with the SD is indicated by the one-sigma error ellipse. The line indicates the projection of the shower axis onto the ground. 


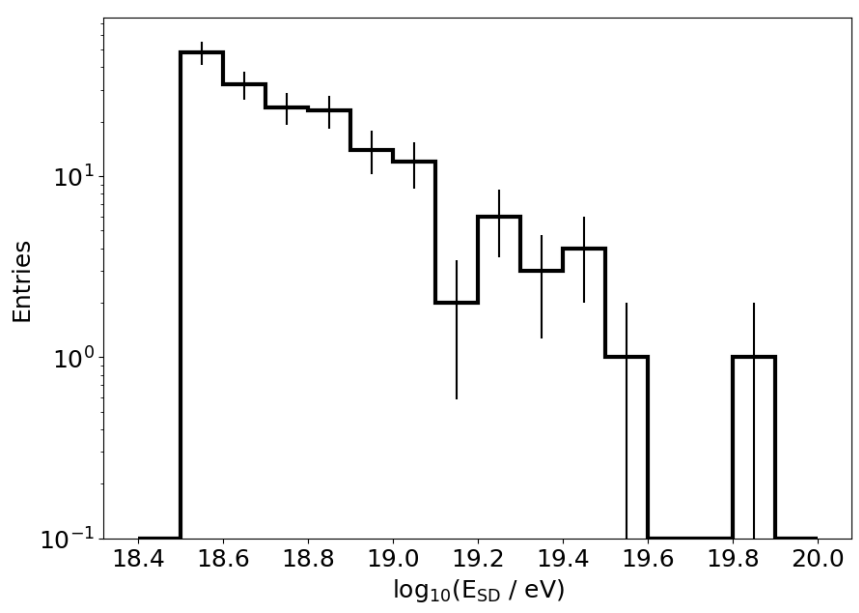

Figure 5: Distribution of the energy reconstructed with the surface detector. For each bin Poissonian errors are shown.
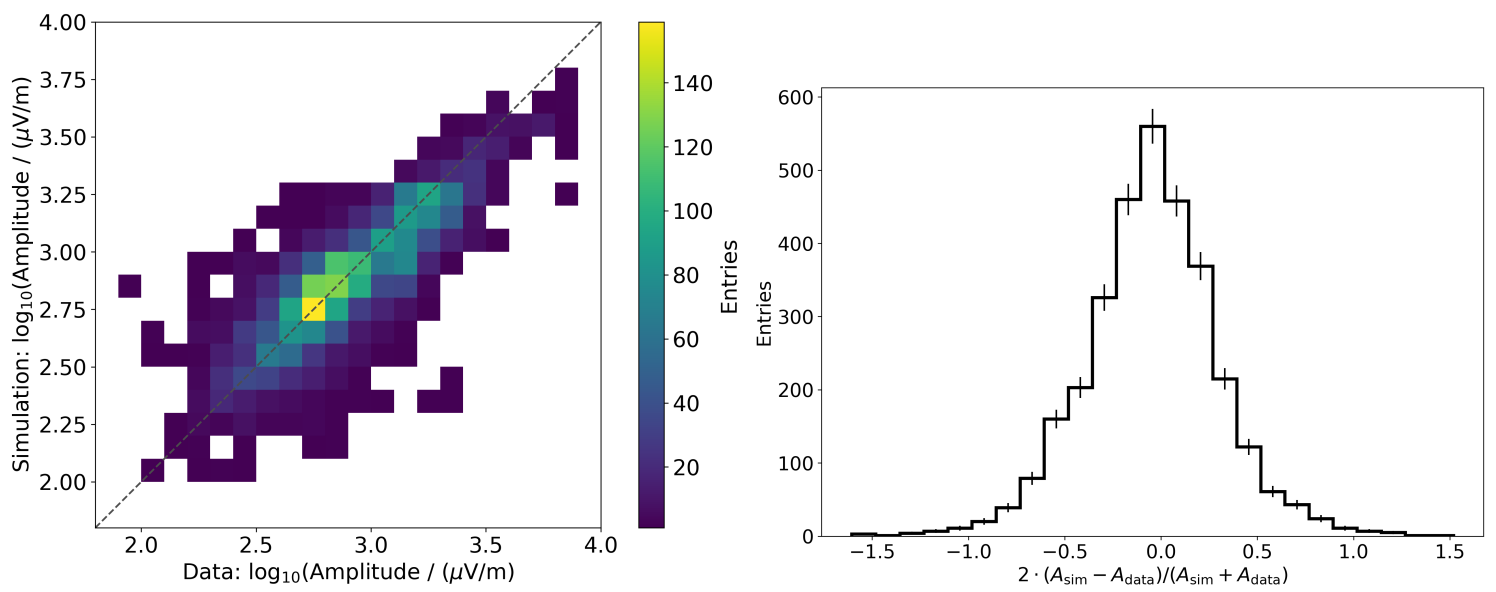

Figure 6: Correlation of the peak amplitudes of measured data and CoREAS simulations for the subset of events with a reconstructed SD energy visualized as the two dimensional histogram and the one dimensional projection.

pulse individually shows a good agreement between data and simulation, see Fig. 6. On average, the simulations underpredict the measured amplitudes by $5 \%$ with a spread of $35 \%$. The large spread is caused by the uncertainty of the reconstructed energy and estimated shower core and will likely improve with a radio reconstruction of inclined air showers.

\subsection{Prospects of a sparse radio grid}

To study the prospects of radio detection using a sparse antenna grid we thin out AERA to an approximate $1.5 \mathrm{~km}$ grid. This results in 5 stations for AERA phase II and 10 stations for AERA phase III (cf. Fig. 1). We rerun the analysis on the same raw data and obtain 189 reconstructed radio events. For each event where the opening angle of particle and radio shower axis calculated with the full AERA is smaller than $2.6^{\circ}$ we determine the their difference for a sparse AERA grid. On average the opening angle increases by only $0.1^{\circ}$ from $0.6^{\circ}$ for AERA to $0.7^{\circ}$ for the sparse grid. 


\section{Challenges for radio reconstruction of inclined air showers}

The radiation energy, i.e. the integral of the lateral distribution function (LDF) of the energy fluence in the form of radio signals, was shown to be a suitable estimator of the primary cosmic ray energy in the case of vertical air showers [10,11]. For inclined air showers two new asymmetries have to be taken into account for a precise reconstruction of the radiation energy arising from the propagation of the radio emission through the atmosphere.

The first asymmetry is introduced by early-late effects, i.e. the fact that signals from "early" part of the shower correspond to a narrower beamed emission than signals from the "late" part. The axis distance of each antenna in the shower plane has to be corrected to match a projection along the line of sight from the position in the ground plane to the source, i.e. we describe the LDF in terms of off-axis angle. This can be corrected to first order by assuming that the radio emission originates from a point source located at a known shower maximum $X_{\max }$ [12].

The second observed asymmetry is related to a difference in the effective refractive index seen by close and far antennas. This results in a Cherenkov compression of the radio emission at a different off-axis angle. We simulate antennas on the positive and negative $\mathbf{v} \times(\mathbf{v} \times \mathbf{B})$ axis ( $\mathbf{v}$ : shower axis, B: magnetic field) and project them onto the ground. On this axis the two different components of the radio emission decouple [13]. Thus we do not have to take interference effects into account. The simulated radio stations are then shifted into the shower plane and the early-late corrections are applied. In Fig. 7 we show the difference in the LDF for an air shower coming from south with a zenith angle of $85^{\circ}$. A clear difference in the peak position is shown that disturbs the radial symmetry assumed internally by all current LDF models. This asymmetry vanishes for simulations where the refractive index is fixed to 1 and increases for larger refractive indices.

A radio reconstruction for inclined air showers that include these asymmetries is currently under investigation [14].

\section{Conclusion}

With AERA, we have measured the radio emission of 1972 air showers with a zenith angle between $60^{\circ}$ and $84^{\circ}$. We have shown that the size of the radio emission footprint increases with the zenith angle and illuminates areas of several $\mathrm{km}^{2}$ on the ground. We have shown that the amplitudes predicted by CoREAS correspond within systematic uncertainties to the measurements. Further work is needed to establish a radio reconstruction of inclined air showers that includes the early late and refractive index asymmetry and to obtain a reliable determination of cosmic ray observables.

Even with a sparse grid of radio antennas with a spacing of $1.5 \mathrm{~km}$ we can detect 189 air showers. The accuracy of the reconstructed shower axis decreases only marginally. The successful detection of these events motivated the recent decision to upgrade each station of the surface detector with an additional radio antenna. The upgrade will increase the geometric area equipped with radio antennas from $17 \mathrm{~km}^{2}$ to $3000 \mathrm{~km}^{2}$, thus enabling the detection of radio emission from cosmic rays at the highest energies [15]. 


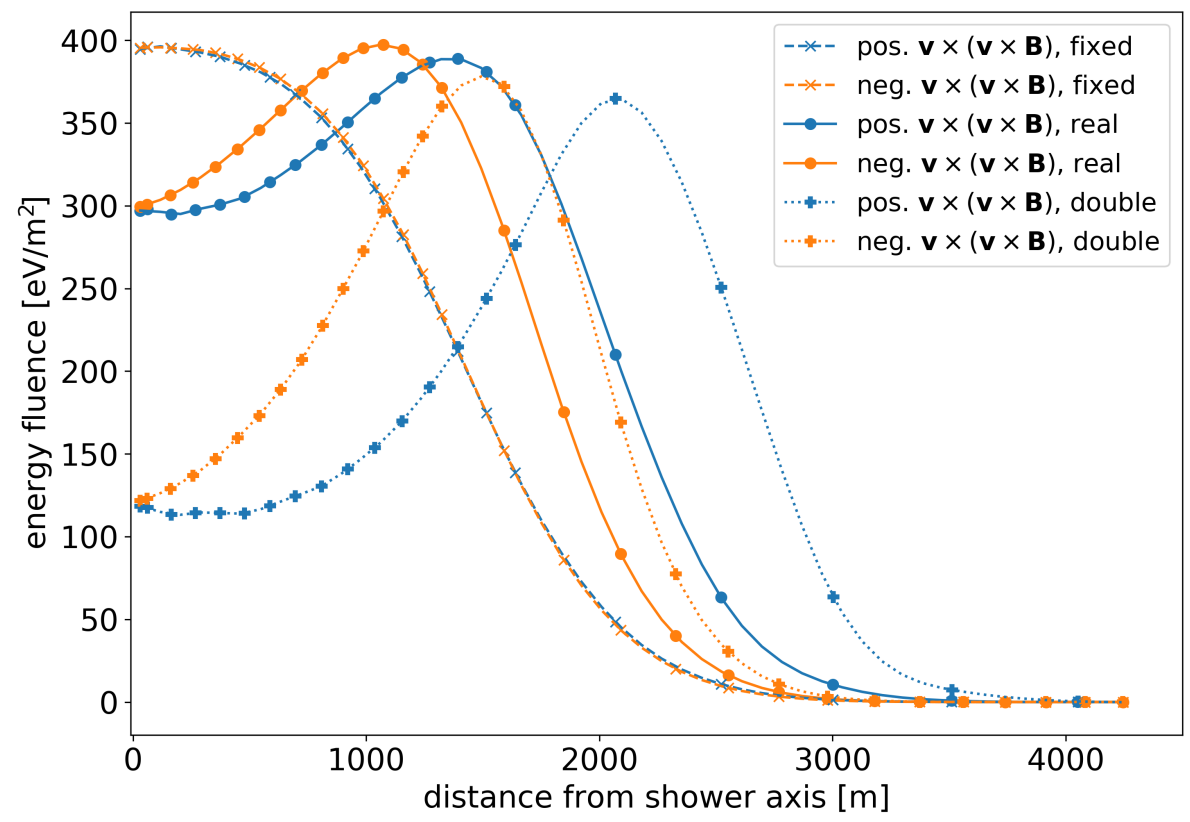

Figure 7: Example of the refractive index asymmetry using different scalings of the refractive index in the CoREAS simulations. We use a constant value of 1 for the whole atmosphere (fixed), a realistic model with a scaling of the refractive index at sea level $\left(n_{0}-1=3.12 \times 10^{-4}\right)$ to higher altitudes (real) and a model where we increased the refractive index at sea level by $1+2 \cdot\left(n_{0}-1\right)$ (double). The fluence values on the positive and negative $\mathbf{v} \times(\mathbf{v} \times \mathbf{B})$ axis are interpolated for convenience.

\section{References}

[1] T. Huege, Physics Reports 620 (2016) 1 -52

[2] F. G. Schröder, Progress in Particle and Nuclear Physics 93 (2017) 1 - 68

[3] T. Huege, A. Haungs, JPS Conf. Proc. 9 (2016) 010018

[4] A.Aab [Pierre Auger Collaboration], Nucl. Instrum. Meth. A 798 (2015) 172

[5] E. M. Holt for the Pierre Auger Collaboration, PoS ( ICRC2 017 ) 492

[6] A.Aab [Pierre Auger Collaboration], JCAP 1810 (2018) 026

[7] T. Huege, M. Ludwig, C. W. James, AIP Conf. Proc. 1535 (2013) 128-132

[8] A.Aab [Pierre Auger Collaboration], JCAP 1408 (2014) 019

[9] H. Dembinski, Ph.D. thesis, RWTH Aachen University (2009), https://web.physik.rwth-aachen.de/ hebbeker/theses/dembinski_phd.pdf

[10] A.Aab [Pierre Auger Collaboration], Phys. Rev. D 93 (2016) 122005

[11] A.Aab [Pierre Auger Collaboration], Phys. Rev. Lett. 116 (2016) 241101

[12] T. Huege, L. Brenk, F. Schlüter, 8th ARENA (2018), [arXiv:1808.00729]

[13] C. Glaser et al., JCAP 1609 (2016) 024

[14] T. Huege, F. Schlüter, L. Brenk, PoS ( ICRC2019) 294

[15] B. Pont for the Pierre Auger Collaboration, PoS (ICRC2019) 395 